\title{
Molecular Analysis of Malassezia Microflora on the Skin of the Patients with Atopic Dermatitis
}

\author{
Seon Mi Yim, M.D. ${ }^{1}$, Ji Young Kim, M.D. ${ }^{1}$, Jong Hyun Ko, M.D. ${ }^{1}$, Yang Won Lee, M.D. ${ }^{1,2}$, \\ Yong Beom Choe, M.D. ${ }^{1,2}$, Kyu Joong Ahn, M.D. ${ }^{1,2}$ \\ Department of Dermatology, ${ }^{1}$ Konkuk University School of Medicine and ${ }^{2}$ Konkuk University Research Institute of Medical Science, \\ Seoul, Korea
}

Background: The yeasts of the genus Malassezia are members of the normal flora on human skin and they are found in $75 \sim 80 \%$ of healthy adults. Since its association with various skin disorders have been known, there have been a growing number of reports that have implicated Malassezia yeast in atopic dermatitis (AD). Objective: The aim of the present study is to isolate the various Malassezia species from AD patients by using 26S rDNA (ribosomal Deoxyribonucleic acid) PCR-RFLP and to investigate the relationship between a positive Malassezia culture and the severity of AD. Methods: Cultures for Malassezia yeasts were taken from the scalp, cheek, chest, arm and thigh of 60 patients with atopic dermatitis. We used a rapid and accurate molecular biological method 26S rDNA PCR-RFLP, and this method can overcome the limits of the morphological and biochemical methods. Results: Positive Malassezia growth was noted on $51.7 \%$ of the patients with atopic dermatitis by 26S rDNA PCR-RFLP analysis. The overall dominant species was $M$. sympodialis $(16.3 \%)$. M. restricta was the most common species on the scalp (30.0\%) and cheek (16.7\%). M. sympodialis $(28.3 \%)$ was the most common species on the chest. The positive culture rate was the highest for the 11 $\sim 20$ age group $(59.0 \%)$ and the scalp showed the highest rate at $66.7 \%$. There was no significant relationship between the Malassezia species and SCORing for Atopic Dermatitis (SCORAD). Conclusion: The fact that the cultured species was different for the atopic dermatitis lesion skin from that of

Received November 11, 2009, Revised November 26, 2009, Accepted for publication December 10, 2009

*This work was supported by Konkuk University in 2009.

Reprint request to: Kyu Joong Ahn, M.D., Department of Dermatology, Konkuk University School of Medicine, Konkuk University Research Institute of Medical Science, 4-12, Hwayang-dong, Gwangjin-gu, Seoul 143-729, Korea. Tel: 82-2-2030-5170, Fax: 82-2-2030-5179, E-mail: kjahn@kuh.ac.kr the normal skin may be due to the disrupted skin barrier function and sensitization of the organism induced by scratching in the AD lesion-skin. But there was no relationship between the Malassezia type and the severity score. The severity score is thought to depend not on the type, but also on the quantity of the yeast. (Ann Dermatol 22(1) $41 \sim 47,2010$ )

-Keywords-

$26 \mathrm{~S}$ rDNA PCR-RFLP, Atopic dermatitis, Malassezia

\section{INTRODUCTION}

The yeasts of the genus Malassezia are members of the normal flora on human skin and they are found in 75 $80 \%$ of healthy adults ${ }^{1}$. The colony-formation begins immediately after the birth, and the population density increases on skin areas that have a high sebum production. Its prevalence is the highest for patients who are in late adolescence and early adulthood ${ }^{2}$. Malassezia yeast was first reported on in 1889, and it has been implicated in various diseases, such as pityriasis versicolor, seborrheic dermatitis and Malassezia folliculitis. Recently, there have been a growing number of reports that have implicated Malassezia yeast in atopic dermatitis ${ }^{2}$.

Atopic dermatitis (AD) is a multifactorial disease in which both hereditary and environmental factors play an important role. Therefore, the Malassezia species in patients with AD serve not only as the normal skin flora, but also as an exacerbating factor. The number of patients with AD, and particularly adults who respond poorly to antiinflammatory treatment, have recently been increasing ${ }^{3}$. Treatment with anti-fungal agents for the patients who are 
affected mainly on the head and neck regions has been found to decrease Malassezia colonization and the severity of $A D$ lesions, which suggests that the Malassezia species play a certain role in $\mathrm{AD}^{4}$.

The genus Malassezia currently consists of 11 species: $M$. dermatis, M. furfur, M. globosa, M. japonica, M. nana, $M$. obtusa, M. pachydermatis, $M$. restricta, M. slooffiae, $M$. sympodialis and $M$. yamatoensis. M. nana and M. pachydermatis show affinity for animals, while the remaining nine species colonize human skin. Several studies have examined which species are involved in exacerbating $\mathrm{AD}^{3,5,6}$. However, the results of culture-based analyses of the cutaneous Malassezia flora in patients with AD have differed among studies. These discrepancies are likely to have occurred due to different isolation techniques, different media and the various growth characteristics of each species.

Therefore, various molecular biological techniques such as direct sequencing, PCR (polymerase chain reaction), RFLP (restriction fragment length polymorphism), RAPD (random amplification of polymorphic DNA), SSCP (single strand conformation polymorphism), DGGE (denaturing gradient gel electrophoresis) and T-RFLP (terminal restriction fragment length polymorphism) are gradually replacing the morphologic method.

The aim of the present study is to isolate the various Malassezia species from AD patients by using 26S rDNA (ribosomal Deoxyribonucleic acid) PCR-RFLP and to investigate the relationship between a positive Malassezia culture and the severity of AD.

\section{MATERIALS AND METHODS}

\section{Patients}

Sixty AD patients at Konkuk University Hospital were enrolled in this study. They were divided into 3 groups according to their age range from 0 to 10,11 to 20 and 21 to 30 . These groups had 10 males and 10 females in each group. The diagnosis of AD was based on the criteria of Hanifin and Rajka ${ }^{7}$. Five body sites were selected for the examination: the scalp, cheek, chest, upper arm and thigh. Most of the body sites were atopic dermatitis involved areas. The patients were instructed not to take a shower or use emollients on the day of investigation. The use of systemic glucocorticoids, systemic antifungal treatment or ultraviolet phototherapy was not allowed for 2 months prior to the investigation. Topical antifungal treatment was not allowed 1 month before the study and topical corticosteroids were not allowed for 1 week before the study. Written informed consent was obtained from each subject before the procedure. The investigations were conducted according to the principles of the Declaration of Helsinki.

\section{Sampling from the skin}

Samples from the skin were taken by the swabbing technique $^{8}$. The detailed procedure was as follows: swabs moistened in $100 \mu \mathrm{l}$ of detergent were rubbed five times against $3 \mathrm{~cm}$ of the skin from the scalp, cheek, chest, upper arm and thigh. Then only the fiber-tipped part of the swab was cut and then placed in $900 \mu$ l of detergent. This was placed into a shaker for 30 seconds to evenly disperse the Malassezia yeasts in the solution. About 100 $\mu$ I of the sampled skin was mixed with $900 \mu$ l of the detergent along with half of the concentration. From this, $100 \mu$ l was taken to be evenly applied on Leeming \& Notman culture medium ${ }^{9}$ and this was incubated at $34^{\circ} \mathrm{C}$ for 14 days. To isolate the cultured Malassezia yeasts, the shape and size of the colony and the changes of the media were observed, and distinct colonies were collected and analyzed.

\section{DNA extraction and PCR}

For the DNA extraction and PCR analysis of the isolates from skin, this study adopted colony PCR analysis, which was developed to extract DNA directly from the colonies of a PCR tube and to amplify the $26 \mathrm{~S}$ rDNA at the same time instead of using the direct genomic DNA extraction methods. A single colony of Malassezia yeast was taken and transferred to a PCR tube and it was warmed up in a double boiler by using a microwave 3 times a day for 1 minute, and then the tube was moved into ice water. The PCR reaction mixture $(0.25 \mathrm{mM}$ deoxynucleoside triphosphate, 10X PCR buffer, 5X Q buffer, $0.5 \mu \mathrm{M}$ primers, $1.25 \mathrm{U}$ Hot StarTaq polymerase, $20 \mathrm{mM} \mathrm{MgSO}_{4}$ ) was added and vortex mixing was done. Then, PCR using a Mastercycler 5333 (Eppendorf, Hamburg, Germany) was immediately performed. To amplify the $26 \mathrm{~S}$ rDNA, the primer that can amplify all 11 standard strains at once was chosen. The sequence was forward, 5'-TAACAAGGATTC CCCTAGTA-3' and reverse, 5'-ATTACGCCAGCATCCTAA $\mathrm{G}-3^{10}$. The conditions in the early stage of the reaction were $95^{\circ} \mathrm{C}$ for 14 minutes for pre-denaturation, $94^{\circ} \mathrm{C}$ for 45 seconds for denaturation, $55^{\circ} \mathrm{C}$ for 45 seconds for annealing, $72^{\circ} \mathrm{C}$ for 1 minute for extension of the 40 cycles, and then $72^{\circ} \mathrm{C}$ for 7 minutes for the last extension. The amplified DNA was visualized by electrophoresis on a $1.5 \%(\mathrm{w} / \mathrm{v})$ agarose gel with using ethidium bromide $(0.5 \mu \mathrm{g} / \mathrm{ml})$ and by using 1 X TAE migrating buffer $(\mathrm{pH}$ 8.0, $40 \mathrm{mM}$ Tri-acetate $1 \mathrm{mM}$ EDTA). 


\section{Restriction fragment length polymorphism (RFLP analysis)}

After confirmation of the amplified 26S rDNA, the PCR products were purified using an Accu-Prep PCR purification kit (Bioneer, Daejeon, Korea). Two restriction enzymes, Hha1 (Takara Biomedicals, Otsu, Japan) and BstF51 (SibEnzyme, Novosibirsk, Russia), were used to perform the $26 \mathrm{~S}$ rDNA-RFLP of Malassezia ${ }^{11}$. In this experiment, the restriction enzyme digestion was performed with 10X PCR buffer, $10 \mathrm{U}$ of the restriction enzyme and $7.5 \mu \mathrm{l}$ of the PCR products, which sum up to $20 \mu \mathrm{l}$. After the reaction at $37^{\circ} \mathrm{C}$ for 3 hours, the electrophoresis was done on $3.5 \%(\mathrm{w} / \mathrm{v})$ NuSieve GTG agarose gel (FMC, Rockland, ME, USA) at 100 volts and staining was done with ethidium bromide. The restriction fragments were analyzed according to the size and number of DNA fragments seen under the UV transilluminator.

\section{Statistical analysis}

Comparision of the AD patient groups was done according to clinical severity as reflected by an SCORAD above or below 40, and Fisher's exact test was used for statistical analysis.
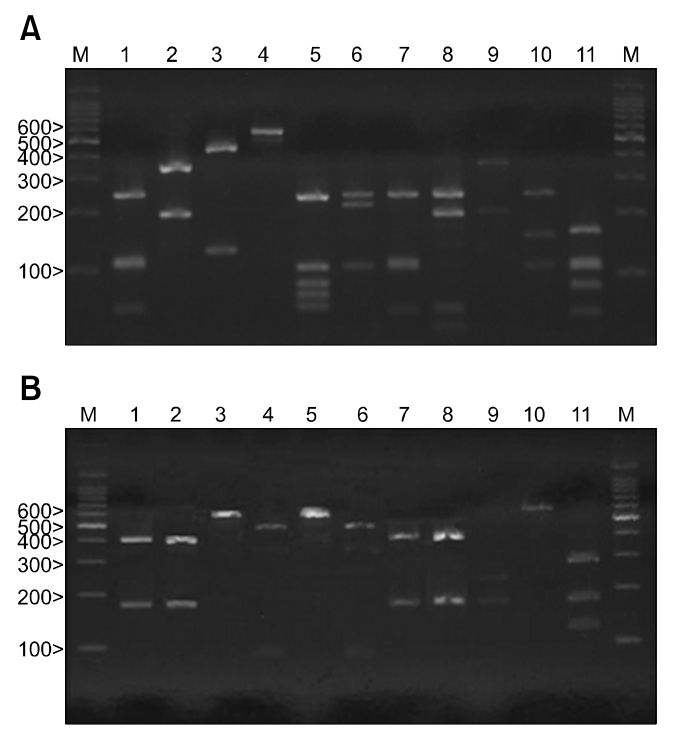

Fig. 1. PCR-RFLP patterns of the $26 \mathrm{~S}$ rDNA PCR digested with Hha1 (A) and BstF51 (B) of the 11 standard strains of Malassezia. Lanes: $M$ means molecular marker, 1: M. furfur (KCTC 7743), 2: M. sympodialis (KCTC 7985), 3: M. globosa (CBS 7966), 4: M. restricta (KCTC 7848), 5: M. slooffiae (KCTC 17431), 6: M. pachydermatis (KCTC 17008), 7: M. japonica (CBS 9432), 8: $M$. nana (JCM 12085), 9: M. dermatis (JCM 11348), 10: M. obtusa (KCTC 7847), 11: M. yamatoensis (CBS 9725).

\section{RESULTS}

\section{S rDNA PCR-RFLP analysis of the Malassezia standard strains}

After the amplification of the 26S rDNA of the Malassezia standard strains, a 580 bp PCR band was seen for all the 11 standard strains. These PCR products were digested with restriction endonucleases Hha1 and BstF51 by agarose gel electrophoresis. M. furfur, M. globosa, $M$. japonica, M. nana, M. obtusa, $M$. pachydermatis, $M$. slooffiae and $M$. yamatoensis were distinguished using Hha1 (Fig. 1A). On the other hand, M. dermatis and $M$.

Table 1. The detection rate of Malassezia species according to the age group in atopic dermatitis patients

\begin{tabular}{cccccc}
\hline \multirow{2}{*}{$\begin{array}{c}\text { Age } \\
\text { groups }\end{array}$} & \multicolumn{4}{c}{ No. of Malassezia species } \\
\cline { 2 - 5 } & Male $(\%)$ & Female $(\%)$ & Total $(\%)$ \\
\hline $0 \sim 10$ & $26 / 50(52.0)$ & $20 / 50(40.0)$ & $46 / 100(46.0)$ \\
$11 \sim 20$ & $39 / 50(78.0)$ & $20 / 50(40.0)$ & $59 / 100$ & $(59.0)$ \\
$21 \sim 30$ & $29 / 50(58.0)$ & $21 / 50(42.0)$ & $50 / 100$ & $(50.0)$ \\
\hline Total & $94 / 150(62.7)$ & $61 / 150(40.7)$ & $155 / 300(51.7)$ \\
\hline
\end{tabular}

Table 2. The detection rate of Malassezia species according to the body sites in atopic dermatitis patients

\begin{tabular}{lccc}
\hline Body site & $\begin{array}{c}\text { Male }(\%) \\
(\mathrm{n}=30)\end{array}$ & $\begin{array}{c}\text { Female }(\%) \\
(\mathrm{n}=30)\end{array}$ & $\begin{array}{c}\text { Total }(\%) \\
(\mathrm{n}=60)\end{array}$ \\
\hline Scalp & $20(66.7)$ & $20(66.7)$ & $40(66.7)$ \\
Cheek & $20(66.7)$ & $16(53.3)$ & $36(60.0)$ \\
Chest & $26(86.7)$ & $13(43.3)$ & $39(65.0)$ \\
Upper arm & $19(63.3)$ & $9(30.0)$ & $28(46.7)$ \\
Thigh & $9(30.0)$ & $3(10.0)$ & $12(20.0)$ \\
\hline Total & $94 / 150(62.7)$ & $61 / 150(40.7)$ & $155 / 300(51.7)$ \\
\hline
\end{tabular}

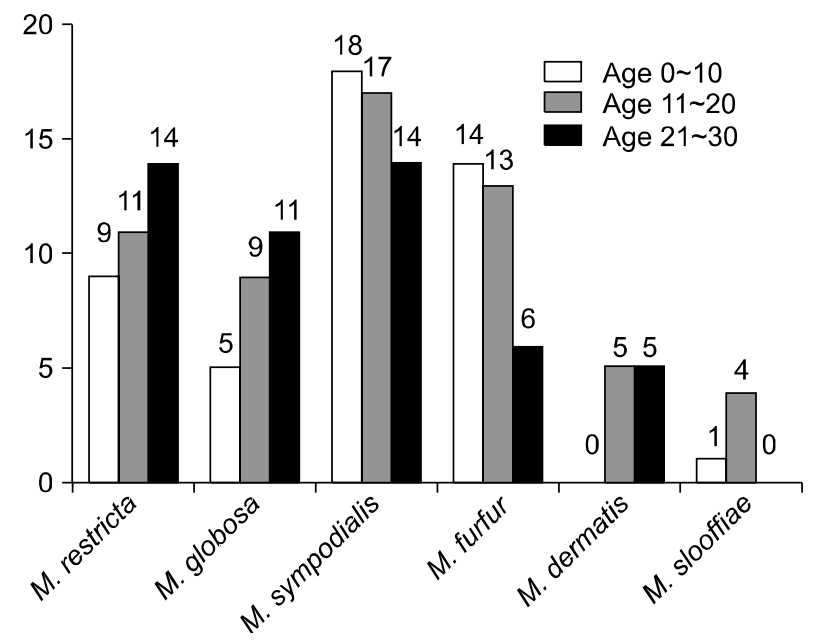

Fig. 2. The identified Malassezia species from atopic dermatitis patients by age. 
sympodialis were distinguished using BstF51 (Fig. 1B). All the Malassezia standard strains could be differentiated by analyzing the 265 rDNA PCR-RFLP pattern with using the two restriction enzymes. The same as the standard strains, all of the colonies that were isolated and cultured from the AD patients were successfully identified using $26 \mathrm{SDNA}$ PCR-RFLP analysis.
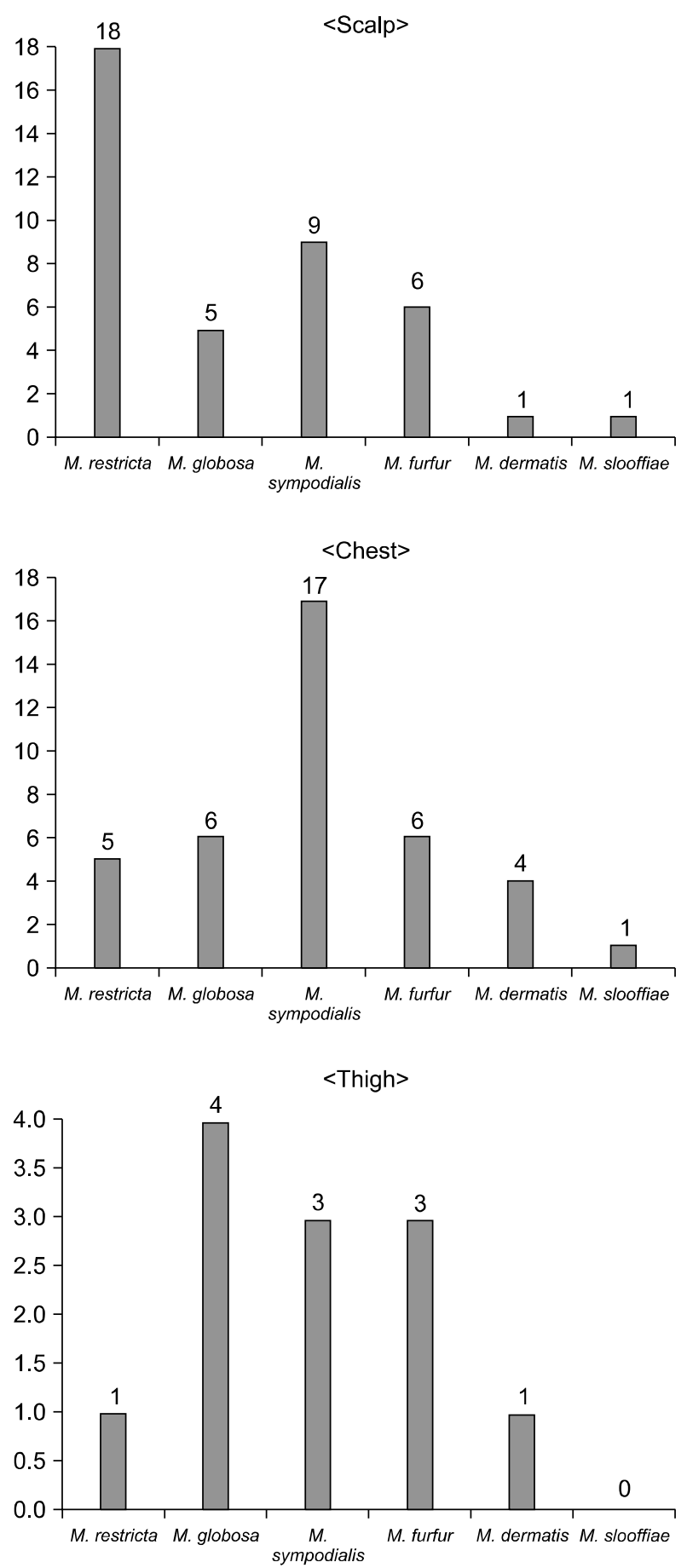

The detection rates of cultured Malassezia yeasts by the different age groups and body sites

Six Malassezia species were identified in the AD patients through the $26 \mathrm{~S}$ rDNA PCR-RFLP method: $M$. restricta, $M$. globosa, M. sympodialis, M. furfur, $M$. dermatis and $M$. slooffiae. The overall positive culture rate of the sampled Malassezia yeasts from the 60 AD patients was $51.7 \%$
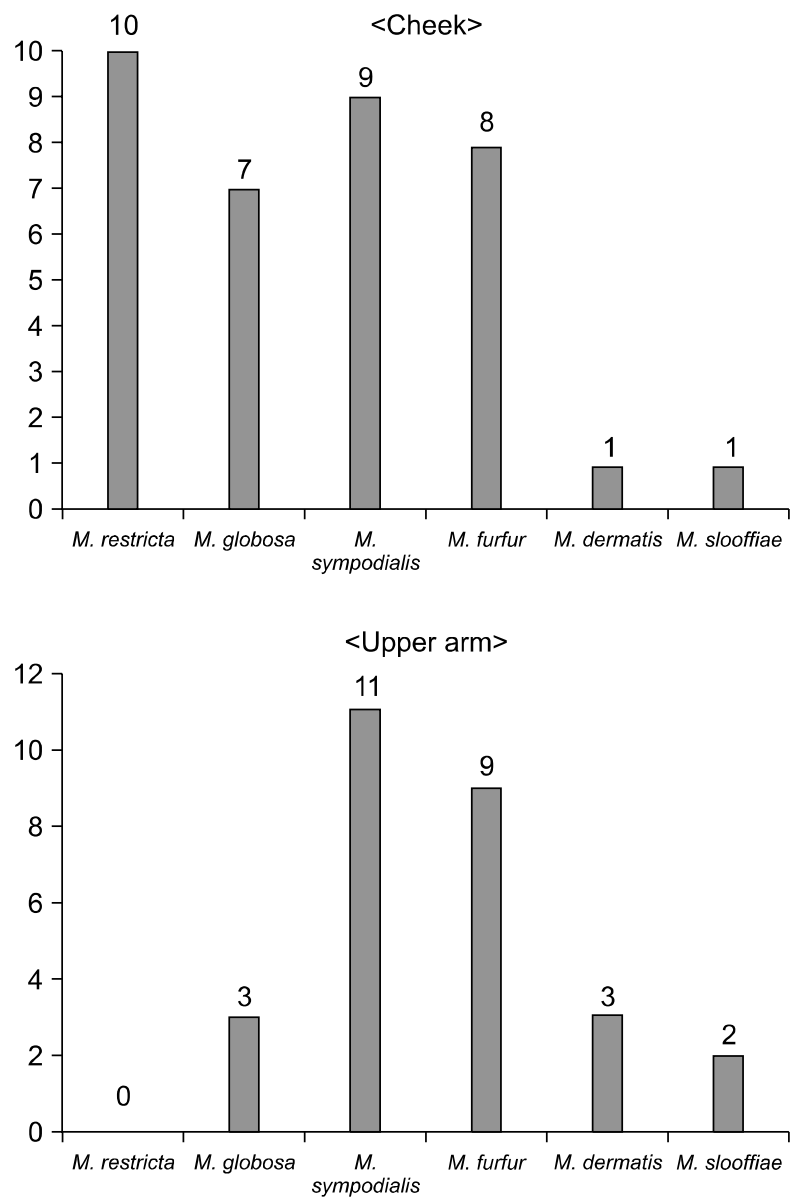

Fig. 3. The identified Malassezia species from the atopic dermatitis groups according to body sites. 
Table 3. Positive Malassezia culture in relation to the severity of atopic dermatitis

\begin{tabular}{llllrlrr}
\hline \multicolumn{1}{c}{ Score } & MR $(\%)$ & MG $(\%)$ & MS $(\%)$ & MF $(\%)$ & MD $(\%)$ & Mslo $(\%)$ & Total $(\%)$ \\
\hline SCORAD $<40$ & $21(20.4)$ & $15(14.6)$ & $33(32.0)$ & $24(23.3)$ & $9(8.7)$ & $1(1)$ & $103(100)$ \\
SCORAD $\geq 40$ & $13(25)$ & $10(19.2)$ & $16(30.8)$ & $8(15.4)$ & $1(1.9)$ & $4(7.7)$ & $52(100)$ \\
\hline Total & $34(21.9)$ & $25(16.1)$ & $49(31.6)$ & $32(20.7)$ & $10(6.5)$ & $5(3.2)$ & $155(100)$ \\
\hline
\end{tabular}

MR: M. restricta, MG: M. globosa, MS: M. sympodialis, MF: M. furfur, MD: M. dermatis, Mslo: M. slooffiae.

(Table 1). For the culture rate by age, the positive culture rate was the highest in the $11 \sim 20$ AG (age group) $(59.0 \%)$. In the experiment groups according to different body areas, the scalp showed the highest positive culture rate at $66.7 \%$ (Table 2).

\section{Molecular biological identification of the Malassezia species by the age groups and body sites using $26 \mathrm{~S}$ rDNA PCR-RFLP analysis}

The results showed that in the AD patients, M. sympodialis was identified most frequently in 49 sites (16.3\%) of the 300 tested sites (Fig. 2). Among the Malassezia yeasts cultured on the different body areas according to the AGs, M. sympodialis was identified as the common species in $18.0 \%$ of the $0 \sim 10 \mathrm{AG}$, followed by $17.0 \%$ in the $11 \sim 20$ AG and $14.0 \%$ in the $21 \sim 30$ AG (Fig. 2). The results from each body site indicated that $M$. restricta was the most common species on the scalp (30.0\%) and cheek $(16.7 \%)$. M. sympodialis $(28.3 \%)$ was the most common species on the chest in the AD patients (Fig. 3).

\section{Positive Malassezia culture in relation to the severity of AD}

For the patients with severe AD (a SCORAD $\geq 40$ ), the Malassezia cultures were positive in 52 of 87 cases $(59.8 \%)$, as compared with 103 of the 213 cases $(48.4 \%)$ with less severe AD (a SCORAD $<40$ ). The Malassezia species that were more commonly cultured from the patients with $\mathrm{AD}$ and a SCORAD $\geq 40$ were $M$. sympodialis in 16 of 52 cases $(30.8 \%), M$. restricta in 13 cases $(25 \%)$ and M. globosa in 10 cases $(19.2 \%)$. The Malassezia species that were more commonly cultured from the patients with $\mathrm{AD}$ with a SCORAD $<40$ were $M$. sympodialis in 33 of 103 cases (32\%), M. furfur in 24 patients $(23.3 \%)$ and $M$. restricta in 21 patients (20.4\%) (Table 3 ). But there were no significant differences between a positive Malassezia culture, the Malassezia species and the severity of $A D$.

\section{DISCUSSION}

According to earlier reports, M. sympodialis, M. globosa and M. furfur were the most commonly isolated Malassezia species from healthy individuals and from patients with diseases like atopic dermatitis ${ }^{11-16}$. M. restricta and M. globosa were the most frequently isolated species in a Korean study ${ }^{17}$. However, there were differences with a respect to the commonly isolated species, not only between normal healthy individuals and the patients with various skin diseases, but also between different countries. For the healthy subjects from a previous study, the dominant species was $M$. globosa ${ }^{18}$, while $M$. sympodialis was the dominant species in a study from Canada ${ }^{12}$. For the patients with $\mathrm{AD}$ in this study, the dominant species was M. sympodialis, while M. furfur ${ }^{15}$ or M. globosa and M. restricta ${ }^{16}$ were the dominant species in Japan and $M$. sympodialis was the dominant species in Canada ${ }^{13}$. This difference may be attributable to the sampling and culture techniques such as our swabbing-culture-PCR-RFLP technique or the Opsite-nonculture-PCR-RFLP, as well as to racial and geographical differences.

The results from each body site indicated that $M$. restricta was the most common species on the scalp (30.0\%) and cheek $(16.7 \%)$. M. sympodialis $(28.3 \%)$ was the most common species of AD patients on the chest (Fig. 3). The detection rate was lower on the thigh and upper arm among the different body sites. There could be a relationship among the prevalence sites of atopic dermatitis, the culture rate and the different isolated species, although this has not been clearly identified.

We understand that several subtypes of Malassezia may coexist on the same individual. We actually have confirmed the isolation of more than one subtype during the culture and PCR-RFLP process. However, in this study, the results were derived from the dominant subtypes (the dense band on PCR-RFLP).

In this study, the prevalence of the Malassezia yeasts was lower for the AD patients as compared to that of the healthy subjects from a previous study ${ }^{18}$. Further proof is needed for our observation that patients with AD harbor a lower number of Malassezia yeasts. One reason for this may be the reduced amount of lipids in the skin of $A D$ patients ${ }^{19}$. In a recent study, the number of yeast cells on patients with $\mathrm{AD}$ was lower in the lesional skin as 
compared to that of the nonlesional skin. In a previous study, cultures from the lesional skin of patients with $A D$ were, for approximately $25 \%$ of the subjects, taken from the arm or leg, which are locations known to harbor Malassezia less often than the trunk, neck and forehead ${ }^{20}$. Another explanation may be the antifungal activity of the mediators and/or inflammatory cells present in AD lesions. According to recent studies, Malassezia may be an important allergen and trigger factor in $A D$, and especially for the AD located in the head and neck region ${ }^{21,22}$. In this current study, the prevalence of positive Malassezia cultures was not higher for the patients with severe AD or a $\mathrm{SCORAD} \geq 40$, as compared with those patients who had a SCORAD $<40$. The role of Malassezia yeasts as a trigger factor in $A D$ is probably due to an allergic reaction. There was no difference between positive cultures of different Malassezia species and the severity of AD. Quantitative analysis like RT-PCR may be needed to clarify the relationship between Malassezia and atopic dermatitis.

From this study, the recovery rate from AD was lower than that of other studies. The reason for this is the limitation of the age group from 0 to 30. Atopic dermatitis is a chronic, fluctuating, inflammatory skin disease that rarely presents in adulthood; it commonly occurs within the first 2 years after the birth. The worldwide prevalence of AD is $10 \sim$ $20 \%$ in children and $1 \sim 3 \%$ in adults ${ }^{23}$. So in this study, we enrolled $A D$ patients from the $0 \sim 30$ age group, and not from the AD patients of all ages. Our result of the distribution of the Malassezia species was similar to that of many previous studies, but the recovery rate was lower. In many recent studies, real time PCR or nested PCR were popularly used for identifying the Malassezia species. However, 26S rDNA PCR RFLP is a genotypic identification approach that can be applied for identifying nearly all known Malassezia species and it is a flexible procedure because patterns of newly described species can be added directly to a database without the need for sequence information. This PCR-RFLP method requires only PCR and one or two enzymes, and it is technically less demanding than most other molecular biological approaches ${ }^{12}$. Lee et al. ${ }^{24}$ previously compared the obtained DNA sequences with the full sequences of the genes already available in GeneBank, and this showed 99\% concordance. Oh et al. $^{25}$ re-identified Malassezia species using nested PCR from the 327 positive samples, with those samples with 2 or more identified yeasts being excluded. As a result, 320 out of the 327 samples (98.8\%) were concordant with the results of the RFLP method. Thus, the 265 rDNA PCRRFLP method for identifying 11 Malassezia species is capable of identifying species with high accuracy and reliability, and it is simple, fast and cost effective for use even in routine laboratories.

In conclusion, we found fewer individuals with positive Malassezia cultures among the AD patients. It was also confirmed that the prevalence of a positive Malassezia culture was not correlated with the severity of AD.

This makes it rather difficult to elucidate the role of Malassezia in atopic dermatitis on the basis of the detection rate alone. The relationship between atopic dermatitis and the Malassezia species should be clarified through further studies.

The species cultured from the AD patients' skin differed from that of the normal skin, as much it varied among different locations of AD lesions. These facts may be due to disrupted skin barrier function and the sensitization to the organism that's induced by scratching in AD lesions. In order to determine the distribution and the character of Malassezia yeasts on the skin of AD patients, more case control studies and quantitative molecular biologic analysis should be done by selecting specific types of subjects from AD patients.

\section{REFERENCES}

1. Ahn KJ. Taxonomy of the genus Malassezia. Korean J Med Mycol 1998;3:81-88.

2. Ljubojevic S, Skerlev M, Lipozencic J, Basta-Juzbasic A. The role of Malassezia furfur in dermatology. Clin Dermatol 2002;20:179-182.

3. Baker BS. The role of microorganisms in atopic dermatitis. Clin Exp Immunol 2006;144:1-9.

4. Nikkels AF, Pierard GE. Framing the future of antifungals in atopic dermatitis. Dermatology 2003;206:398-400.

5. Faergemann J. Atopic dermatitis and fungi. Clin Microbiol Rev 2002;15:545-563.

6. Aspres N, Anderson C. Malassezia yeasts in the pathogenesis of atopic dermatitis. Australas J Dermatol 2004;45:199-207.

7. Hanifin JM, Rajka G. Diagnostic features of atopic dermatitis. Acta Derm Venereol 1980;92(Suppl):44-47.

8. Leeming JP, Notman FH, Holland KT. The distribution and ecology of Malassezia furfur and cutaneous bacteria on human skin. J Appl Bacteriol 1989;67:47-52.

9. Leeming JP, Notman FH. Improved methods for isolation and enumeration of Malassezia furfur from human skin. J Clin Microbiol 1987;25:2017-2019.

10. Mirhendi H, Makimura K, Zomorodian K, Yamada T, Sugita T, Yamaguchi H. A simple PCR-RFLP method for identification and differentiation of 11 Malassezia species. J Microbiol Methods 2005;61:281-284.

11. Crespo Erchiga V, Ojeda Martos A, Vera Casano A, Crespo Erchiga A, Sanchez Fajardo F. Malassezia globosa as the causative agent of pityriasis versicolor. Br J Dermatol 2000; 143:799-803.

12. Gupta AK, Kohli Y, Faergemann J, Summerbell RC. Epidemiology of Malassezia yeasts associated with pityriasis 
versicolor in Ontario, Canada. Med Mycol 2001;39:199206.

13. Gupta AK, Kohli Y, Summerbell RC, Faergemann J. Quantitative culture of Malassezia species from different body sites of individuals with or without dermatoses. Med Mycol 2001;39:243-251.

14. Kim SC, Kim HU. The distribution of Malassezia species on the normal human skin according to body region. Korean J Med Mycol 2000;5:120-128.

15. Nakabayashi A, Sei Y, Guillot J. Identification of Malassezia species isolated from patients with seborrhoeic dermatitis, atopic dermatitis, pityriasis versicolor and normal subjects. Med Mycol 2000;38:337-341.

16. Sugita $T$, Suto $H$, Unno $T$, Tsuboi $R$, Ogawa $H$, Shinoda $T$, et al. Molecular analysis of Malassezia microflora on the skin of atopic dermatitis patients and healthy subjects. J Clin Microbiol 2001;39:3486-3490.

17. Lee YW, Yim SM, Lim SH, Choe YB, Ahn KJ. Quantitative investigation on the distribution of Malassezia species on healthy human skin in Korea. Mycoses 2006;49:405-410.

18. Jang SJ, Lim SH, Ko JH, Oh BH, Kim SM, Song YC, et al. The investigation on the distribution of Malassezia yeasts on the normal Korean skin by $26 \mathrm{~S}$ rDNA PCR-RFLP. Ann Dermatol 2009;21:18-26.

19. Schafer L, Kragballe K. Abnormalities in epidermal lipid metabolism in patients with atopic dermatitis. J Invest Dermatol 1991;96:10-15.

20. Sandstrom Falk $M H$, Tengvall Linder $M$, Johansson $C$, Bartosik J, Back O, Sarnhult T, et al. The prevalence of Malassezia yeasts in patients with atopic dermatitis, seborrhoeic dermatitis and healthy controls. Acta Derm Venereol 2005;85:17-23.

21. Sugita $T$, Takashima $M$, Shinoda $T$, Suto $H$, Unno $T$, Tsuboi $R$, et al. New yeast species, Malassezia dermatis, isolated from patients with atopic dermatitis. J Clin Microbiol 2002;40:1363-1367.

22. Scheynius A, Johansson C, Buentke E, Zargari A, Linder MT. Atopic eczema/dermatitis syndrome and Malassezia. Int Arch Allergy Immunol 2002;127:161-169.

23. Takahata $Y$, Sugita T, Kato H, Nishikawa A, Hiruma M, Muto M. Cutaneous Malassezia flora in atopic dermatitis differs between adults and children. Br J Dermatol 2007;157:11781182.

24. Lee YW, Lim SH, Ahn KJ. The application of $26 \mathrm{~S}$ rDNA PCR-RFLP in the identification and classification of Malassezia yeast. Korean J Med Mycol 2006;11:141-153.

25. Oh BH, Song YC, Lee YW, Choe YB, Ahn KJ. Comparison of nested PCR and RFLP for identification and classification of Malassezia yeasts from healthy human skin. Ann Dermatol 2009;21:352-357. 